\title{
1. Communication in development and social change: a genealogy of the field
}

\author{
Srinivas Raj Melkote and Arvind Singhal
}

The need for development of human societies and concomitant modernization initiatives have been around for a long time. In its modern avatar, directed development of human societies dates to the decades immediately following World War II when the task of getting Western European countries back on their feet was paramount. Additionally, this period also witnessed the political freedom accorded to most of the European colonies in Asia, Africa and the Caribbean, and the birth of the United Nations (UN) and its various agencies dealing directly with issues of directed development and progressive social change. The genesis of the field of development communication (devcom) began with (a) theories and models in economics, sociology, psychology and political science emanating from West European and North American countries that prescribed and outlined the trajectory of development and social change for the so-called Third World nations, and (b) organized development assistance programs initiated by multilateral and bilateral agencies of the UN and the rich countries in response to the humanitarian crises following World War II and decolonization of Asia, Africa and the Caribbean regions.

\section{BRIEF HISTORY OF DEVCOM}

Devcom has evolved according to the theories/models during each historical period since the mid-twentieth century and the overarching goals of the development programs, starting with the inception of development as a strategic goal of the state, particularly in the newly liberated European colonies in much of Asia, Africa and the Caribbean. An interdisciplinary approach has transformed the research and practice of communication for development and social change during its evolution. The paradigms, theories, models, methodologies and outcomes of development as well as of devcom have evolved and changed in the last $70+$ years.

\section{Modernization Model in Development}

The history of devcom research and practice began with the post-World War II years when Western-style modernization was a preferred development model for countries all over the world. The enterprise of modernization, in which devcom was ensconced, was influenced by the quantitative and empirical social sciences epistemology, theory and methodology; in particular, it had a strong orientation in economics. Important indicators of development were the gross national product (GNP), per capita incomes and other economic indices in countries around the world. In general, devcom interventions were tasked with the responsibility of preparing individuals in developing countries ${ }^{1}$ toward modernization by tweaking their individual attitudes and cultural mores. Mass media, such as the radio, were used in top-down models 


\section{Handbook of communication and development}

of communication to diffuse modernizing and Westernized innovations to the people in the developing countries. Communication was hypothesized as the missing link in the development chain and it was considered the task of the mass media and devcom to inform and educate the masses in the developing countries. Communication models tended to be linear, one-way, top-down and prescriptive from the change agencies to the people. Scholars have dubbed this as the "transmission model of communication."

Many devcom models and programmatic interventions that are influenced by modernization tenets, diffusion of innovations research, social marketing approaches, entertainment education pedagogy and biomedical approaches in health use a behavior change communication model, which is influenced by Western functionalistic sociology and anchored in positivist philosophy and methodology. This positivistic epistemology is characterized by technical and instrumental processes to achieve tangible ends and generate measurable outcomes. The rules and methods of the quantitative empirical social sciences are applied and the outcomes of development and change are mostly quantitative, such as indicators of the economy (for example, the GNP), or in the health-related areas they deal with statistics about rates of infant mortality, maternal deaths, life expectancy and so on. Predictability and generalization of specific results to a wider context are valued.

\section{Participatory Approaches in Development}

Much of this began to change, starting in the 1970s. The concept of development expanded to include many more types of change guided by different paradigms, theories, disciplinary influences, geographical considerations and methodologies. Development was viewed as a widely participatory process of social change and included a society's social and cultural aspects besides the economic. This was the first major interdisciplinary encounter in devcom, bringing other disciplinary tenets as well as epistemologies to the study of development. Importantly, it brought together the positivist and the interpretive paradigms - the epistemologies of the modernization and participatory theories, respectively.

Participatory development approaches are influenced by the "social change communication model" based on the interpretive epistemology and methodologies. The emphasis is on understanding of people, groups and other phenomena within specific contexts and involves thick description, empathy and self-reflection in practical situations (Kemmis, 2006). The rules and conventions of the interpretive method are considered more important and the outcomes of change include many non-quantitative dimensions such as independence, cultural and personal growth, partnership, participation and empowerment.

The different disciplinary and methodological imperatives of the positivist and interpretive epistemologies posed a challenge to the identity of devcom theory and practice. Many observers contend that it changed the way communication was conceptualized and used in development and change-related work. Behavior change communication models of the past were now complemented with newer communication for social change models. In these newer models, a participatory communication approach between the sender and receiver communication structure was used and the bias was toward horizontal participation (subject to subject), critical awareness and dialogic communication processes.

Both the behavior change and the social change models are useful for different objectives and in different contexts. Media information campaigns or strategic health communication programs would be best served by the behavior change communication framework as invoked 
in the communication for modernization models, while social change programs that involve participation, collaboration, capacity building and empowerment are better informed by communication for social change model. While the participatory mode of communication for development (C4D) programs and activities has been a welcome addition to the devcom toolbox, the definitions of participation reflect a wide variety of approaches. In many contexts, the levels of participation required are low and perfunctory. Importantly, participation initiatives in institutional, government and other such development programs are slanted toward maintaining the status quo in society and are seldom counter hegemonic.

\section{Empowerment Approaches in Development}

The concept and practice of empowerment expanded upon the earlier objective of participation in devcom models and practices toward the end of the 1980s. This was the second major interdisciplinary thrust in devcom research and practice. The construct of empowerment identifies the underlying constraints in directed social change such as, for example, the lack of power among the people at the receiving end of development programs. This has brought critical research to the fore. Critical research goes beyond the instrumental interest of achieving and improving development outcomes as in the positivistic method or the self-understanding and interpretive dimensions of the hermeneutic method to also critique the socioeconomic and structural impediments preventing people from emancipation due to discriminative and coercive structures and influences. Disciplines such as community organization, critical education, women's and gender studies and community psychology, among others, now offered concepts and practices that could be readily incorporated in devcom models and programs. While the concept of empowerment was frequently referenced in the disciplines noted above, it was missing or inadequately explicated in devcom, which was mainly influenced by economics, sociology and psychology-related factors in the modernization model of development.

The differences between the modernization and the participatory/empowerment approaches are stark when it comes to their implications for devcom. Modernization utilizes the transmission model in which the communication process involves sending a message through some channel; the process is usually linear and top-down, while the messages are prescriptive and technical in nature. The transmission approach or the delivery of information in the modernization approach is insufficient to the task of development and change. Participation and empowerment objectives require building understanding, empathy, partnerships and capacity with and of the people who constitute the beneficiaries of development programs. The emphasis of devcom work, therefore, has expanded to also value communication for its organizing value besides the sole preoccupation with transmission of information.

The newer disciplinary and methodological imperatives of empowerment therefore posed a challenge to the identity and practice of devcom. It changed the way communication was conceptualized and used in development and change-related work. Development communication in an empowerment paradigm has the goal of empowerment of the people, building of local capacity and equity. The objectives of devcom activities are now expanded to include the activation and the sustainability of social support systems, social networks, empowerment of local narratives, facilitation of critical awareness and facilitation of community power. The role of the devcom worker has moved from being just a communication expert to a collaborator, facilitator, participant, an advocate for individuals and communities, a risk-taker and even an activist on behalf of the people. In summary, the multidisciplinary influences on devcom 


\section{Handbook of communication and development}

have been profound. It has evolved from the role of "communication for development" to "communication in social change." With the broader mandate given to communication, that is, from being a tool for directed change to being considered as the sine qua non of cultural and social processes in human societies, the field is getting greater visibility among a wider range of scholars in disciplines ranging from deliberative democracy to community psychology.

\section{BOX 1.1 INTEGRATION OF BEHAVIOR CHANGE COMMUNICATION AND COMMUNICATION FOR SOCIAL CHANGE APPROACHES}

An example of how behavior change communication models may be integrated and complemented with communication for social change models is provided by Mali's Dioro Child Survival Project ${ }^{2}$ to reduce infant and maternal mortality. Participatory culture-centered research with the target audience showed that Malian culture was both an ally and a foe of the project's goals. On the one hand, the cultural ideal for a Malian husband was to be supportive of his wife during pregnancy. On the other hand, Malian culture proscribed discussion between husband and wife about a pregnancy. Because of the embarrassment associated with broaching the issue, Malian women often did not receive extra care from their husbands nor extra nutrition during pregnancy. This absence of communication during pregnancy contributed to Mali having one of the highest infant and maternal mortality rates in the world.

Dioro Project officials employed three traditional communication channels to overcome this cultural barrier:

- a pendelu, a short cloth undergarment worn by married women, which as a non-verbal and visual cue symbolizes Malian marital roles, spousal duties and privileges,

- griots, who in Malian culture represent oral historians, praise singers, social mediators, entertainers and educators, and

- folk songs to promote improved maternal and child health, encourage communication between husband and wife and increase pregnant women's utilization of healthcare services.

Based on the engagement and involvement of all stakeholders, the communication campaign centered on creating a new visual symbol to represent pregnancy. When pregnant women changed their traditional white pendelu into a green pendelu, husbands could then play their idealized roles as protectors and supporters of their wives. Accordingly, the griots' songs encouraged women to wear green pendelus during pregnancy, while making their husbands aware of this symbolic visual cue and their accompanying spousal responsibilities.

Three months after launching the initial campaign in seven communities in 1989, spousal communication about pregnancy and maternal health rose from 3 percent to 66 percent of all spouses. Nearly 90 percent of husbands, 80 percent of wives and 77 percent of mothers-in-law reported knowing that the green pendelu represented pregnancy. When husbands were asked what they would do if their wives wore the green pendelu, 42 percent said they would immediately lighten their wife's workload and 50 percent said they would ensure that they received good nutrition. Discussions of pregnancy were no longer taboo in the seven communities in which the green pendelu campaign was conducted. 
The Malian case illustrates that for health projects to be effective, the cultural values, beliefs and practices of the targeted community need to be thoroughly understood. This could only happen when social change programs involved participation, collaboration and a deep understanding of the local culture, symbols and relational practices.

\section{Social Justice Imperatives in Development}

In the last 70+ years since development was adopted as an important goal by the UN and several bilateral and multilateral state agencies, the process of directed development has benefitted many people and communities; their standards of living and consumption of critical resources have risen. However, empirical accounts show that its spread has been uneven leaving many countries, communities and individuals lagging (Piketty, 2014).

At the dawn of the twenty-first century, the neoliberal paradigm of directed change, influenced by a policy regime dominated by global market-based and supply-side economic theories has brought new challenges to progressive change. In the last 45 years, the rise in income inequality and wealth have seen a dramatic increase globally (The Economist, 2012; Stiglitz, 2012; Piketty, 2014). The relative state of countries today and the unequal stratification of communities and individuals on basic human development indices are indicators of a process of development that has been very uneven and many observers and empirical accounts posit that it has failed a large section of people and communities around the globe. The articulation by the UN of the Millennium Development Goals (MDGs) in 2000 and the Sustainable Development Goals (SDGs) in 2015 is a testament to the stark differences among global communities and individuals even in areas basic to human survival and wellbeing.

What should be the priorities of devcom in the face of contemporary realities and challenges? How can devcom play a useful role to address and counter inequality and injustice in development and social change? What are the ways in which we can visualize and reconstruct and/or expand the mission and roles of media and communication in development and social change toward social justice outcomes? These leading questions posed by scholars, administrators and practitioners are pointing toward social justice imperatives in development and social change.

Increasingly, the idea of development toward social justice outcomes is finding common ground among scholars and professionals. We agree with this view and conceptualize development as a process of directed social change with the articulation of social justice as an important objective. The goal of social justice should serve as an anchor for devcom theory and practice. However, while the term "social justice" connotes a common understanding in the conceptual domain, its operationalization has been a challenge. An important reason why unequal and discriminatory development initiatives prevail is because the operationalization of development and change activities has not reflected all facets of social justice. The chapters in this Handbook, therefore, attempt to capture the different facets of social justice in the roles of media and communication in development and social change. 


\section{ORGANIZATION OF THE HANDBOOK}

The contributors to this book examine communicative actions in development and social change as a heuristic device for a holistic understanding of individuals, communities and cultures as vehicles for change and as agents of social and political action at the micro and macro levels. The chapters reflect the current concerns with inequality plaguing our societies and deal with social mobilization, media mobilization, community mobilization, advocacy communication, participatory communication, empowerment-related communication, capacity-building communication, resistance communication, culture-centered communication and other allied modes for achieving social change with social justice serving as an important anchor.

\section{Part I: Broad Conceptual, Theoretical, Methodological and Programmatic Issues}

The Handbook is divided into three parts. In the first part, the chapters deal with broad conceptual, theoretical, methodological and programmatic issues of interest to scholars, practitioners and administrators in the field of communication for development and social change (CDSC). ${ }^{3}$ Elesha Ruminski, Justin Reedy and Laura Black start off this part in Chapter 2 by examining the concept and practice of deliberative democracy. They view it as a necessary framework for development and social change, which is anchored in social justice values. They provide an overview of deliberative democracy theory and make a case for how social and communication capital, opportunities for public dialogue and deliberation can establish viable communicative norms to provide citizens with choices and opportunities for social-justice-oriented civic engagement that could lead to progressive social change. They stress the roles of public dialogue, deliberation and civic media to constitute a deliberative democracy that supports social justice to all its citizens.

In Chapter 3, Tom Jacobson argues that Amartya Sen's 'capabilities approach' to development should be employed as an overarching conceptual framework for studies of CDSC. He presents us with two justifications. First, the capabilities approach offers CDSC researchers a definition of development, which is interdisciplinary, cosmopolitan and oriented to public communication. The author states that this approach resembles the scope of modernization theory but is free of modernization theory's dichotomous thinking, neoliberal assumptions and ethnocentrism. Second, Jacobson posits that CDSC research can contribute to the capability approach's analysis of communication. In this chapter, he reviews key capabilities concepts and illustrates their relevance to the capabilities approach of CDSC theory and research, as well as a concern for social justice.

In Chapter 4, Aman Luthra and Clayton Rosati examine emerging issues in post-development scholarship and devcom with an emphasis on social justice. They posit that post-development is an important theoretical response to the failures of the dominant paradigm and critique the post-development literature for its lack of alternatives offered in the present era of globalization. They look critically at three areas within post-development theorization, which also resonate with the literature in devcom: place, power and networks. They take up the issue of the marginalization and erasure of physical places that theories of globalization have created by equating the global with power and capital while the local place is bound to labor and tradition. The authors argue that post-development theorists celebrate social movements that are often place-based and local despite criticisms of essentialism and romanticization inherent in such theorization. Further, they argue that the anchoring of place in ethno-territorialism 
poses a potential concern of regressive localism. Also, places are far from immune to capitalist poverty and inequality and in fact participate in the reproduction of capitalist logics. Turning their gaze to devcom theory, Luthra and Rosati observe that in the scholarship of communication for social change, the decolonization of communication research should not accept proto-fascism or narrowly focused nationalist "identitarianism" in exchange for a vague methodology of inclusion. Additionally, the authors argue that post-development postulates must acknowledge and incorporate existing popular movements around the world that demand normative standards of wellbeing of individuals and communities as a specific kind of universal. They caution that the post-development turn should pay attention to these universal forces in their imagination of a pluriverse.

Srinivas Melkote and Leslie Steeves (Chapter 5) emphasize the importance of co-creating communicative equality and furthering social and political action by the subaltern for emancipation from unequal structures. The authors describe the participatory action research (PAR) approach, a well-known theory cum practice in CDSC. PAR is critical research guided by an empancipatory interest and committed to social transformation for social justice. It incorporates a praxis that involves endogenous knowledge generation, debate, social and political action with the aim of recapturing and empowering subaltern knowledge structures. In this chapter, the authors present the scholarship in PAR within the broad field of CDSC by tracing its evolution since the mid-twentieth century, with a detailed discussion of PAR and its praxis within the empowerment paradigm in devcom studies. The authors explicate the PAR methodology and praxis, outline guiding tenets for PAR, describe the roles of development communication workers in empowerment/PAR process and provide examples of PAR.

In Chapter 6, Mohan Dutta, Pooja Jayan and Christine Elers elaborate on the culture-centered approach (CCA) that shares the ideology and practice of PAR. The authors review the CCA literature on social change communication, examining its theoretical roots, presenting the key methodological tenets of the approach and then delving into questions of communication practice within the context of social change. The chapter summarizes key contemporary debates and innovations in the CCA and lays the groundwork for experimentations with the framework in scholarship and practice. The authors posit that the delineation between theory and practice framing much of the literature in CDSC sets up a false dichotomy that does not really grapple with the lived realities of the marginalized. For culture-centered theorizing, the work is embedded in and emerges from the struggles of the subaltern margins against the politics of extraction, expulsion and displacement that target subaltern bodies. The authors offer lessons on co-creating communicative equality and building socialist politics that emerge from these struggles.

In Chapter 7, Lisa Servaes and Jan Servaes present an overview of participatory communication for social change (PCSC), a ubiquitous framework in CDSC. Participatory communication stresses the importance of cultural identity of local communities and democratization and participation at all levels - international, national, local and individual. After positioning PCSC in the broader field of communication approaches related to modernization and development and in the context of sustainability, they argue that participatory communication must start with the local community. It is at the community level that the problems of living conditions are discussed and interactions with other communities are elicited. Though multiple definitions and interpretations of PCSC are found in the CDSC literature, the authors posit that the theoretical work of Paulo Freire and the practical work of the United Nations Educational Scientific and Cultural Organization (UNESCO) effectively encapsulate the objectives and 
aspirations of PCSC. They reiterate that these concepts and practices are important and should be widely accepted as a normative theory of participatory communication. The authors warn that one of the fundamental hindrances to deciding on a participation strategy in development and social change is that it threatens existing hierarchies in societies. However, they caution that participation does not imply that there is no longer a role for specialists, planners and institutional leaders. It only means that the viewpoints of the local people are considered before the resources for projects are allocated and that their suggestions for changes in development policies are given utmost importance. In this chapter, the authors summarize the various participatory approaches and types of participatory communication in use including the role of change agents or facilitators in the process of development and social change.

In Chapter 8, Elske van de Fliert also addresses participatory communication in development and social change but focuses on the crucial factor of sustainability. This chapter provides a review of what is required to make the transformational change necessary to address the complex development issues relating to environment and sustainable development and how participatory communication could play a role in this endeavor. Sustainability has been a guiding principle in development for long but gained renewed impetus through the worldwide adoption of the UN's SDGs following the increasingly tangible environmental and social effects of climate change.

Van de Fliert states that achieving sustainable development, however, has proved complicated, as it requires dealing with complex issues that cut across disciplines, sectors and stakeholder interests. The author explores what it takes to facilitate sustainable development and social change by unravelling the elements of a communication strategy that can bridge these complexities. Van de Fliert informs us that such a strategy is built on the principles of participation and empowerment and applies both "telling" and "sharing" functions of communication to inform, engage and connect stakeholders. The author illustrates these principles and practices by describing two cases where conflicting interests existed between environmental and livelihood objectives. Another lesson learned from these case studies is that participatory communication in support of sustainable development should not be a project on its own, but rather a function embedded in all stages of design, planning, implementation and evaluation of an initiative.

In Chapter 9, Arvind Singhal, Monique Sternin, Shafique Muhammad and Lucía Durá describe and analyse an alternative communication and social change praxis called positive deviance (PD). Contrary to the diffusion of innovations approach in C4D literature, which established that exogenous knowledge and information flowing into a community has the ability to transform the mindset and practices of the local people to accept modernizing innovations, the PD approach instead is based on identifying, amplifying and assessing problem-solving endogenous wisdom that is usually hidden within a community. The PD approach is premised on the observation that in every community there are certain individuals or groups whose uncommon wisdom, behaviors and strategies enable them to find better solutions to problems than their peers, while having access to the same resources and facing worse challenges. "Positive deviants," the authors state, "against overwhelming odds, find ways to solve problems in a more effective manner than their peers." The PD approach embodies several facets of what constitutes actionable wisdom in communication and social change praxis. It looks at marginalized and vulnerable populations as reservoirs of wisdom and agency; importantly, it is a bottom-up flow of innovations in a community. In contrast to the element of "push" in persuasion employed in expert-driven exogenous interventions, the PD 
approach involves more of a "pull" strategy, acting on those who choose to willingly engage, seek, imagine, create and generate value to a community in the process of development and social change.

In Chapter 10, Karin Wilkins and Michael Kim provide a comprehensive analysis of media advocacy for progressive social change, building toward an argument for a holistic framework that locates social justice as central to advocacy communication. They posit that media advocacy is the strategic use of media to advance social and public policy initiatives and promote public health and wellbeing. As such, media advocacy engages media both as a resource toward social change and as an intermediate focus through strategic interventions in policy initiatives for development and change with social justice. However, Wilkins and Kim contend that media advocacy faces several challenges in practice, as it is often inhibited by the political and economic contexts, and in theory, through a conceptualization of participatory governance as elitist. Recognizing these challenges, the authors propose a more comprehensive, yet reflective approach of "advocacy communication" for social justice, not limited to the categorical understanding of different communication for social change strategies in the C4D literature. By examining different cases of advocacy communication and relying on the twin development freedoms outlined by Amartya Sen (2000), the authors demonstrate how advocacy must articulate the connections across "freedom to" and "freedom from" in ways that privilege agency in inclusion and representation.

In Chapter 11, Vinod Pavarala and Kanchan Malik examine the role played by community radio in social change through the lens of participatory communication and locate it within the context of the globalization of media. The authors posit that at present, even though there are multiple media outlets, much of the grammar of creating content feeds into the production values of a globalized marketplace and to an oligopolistic control of media by big multinational companies, resulting in a democracy deficit. The authors consider democratic media such as community radio as a means of inclusion of marginalized people as major stakeholders in the process of social change. In this chapter the authors argue, through a conceptual and empirical survey of community radio in many countries around the world, that to build a robust civil society that can effectively negotiate with those in power for inclusive development and sustainable social change, it is necessary to create decentralized and democratic discursive spaces that promote freedom of expression and equitable access to media. In their view, community radio is one such institutional space that has been effectively used by historically marginalized groups to make their voices heard.

Paolo Mefalopulos, in Chapter 12, argues that though historically C4D's main function has been to promote behavior change that could support improved living conditions, past theoretical models and approaches in C4D have demonstrated their inadequacy in achieving the intended results. In this chapter, the author presents an innovative model aimed at promoting sustainable change to improving people's life, which includes the elements of the Communication-Based Assessment (CBA) and Communication Needs Assessment (CNA) frameworks. The model, named Multidimensional Model for Change (MMC), is based on a mix of theories of change management and the current conception of C4D applied to investigate and understand the larger development picture, which includes the socioeconomic, political and cultural environments besides the communication component. According to Mefalopulos, this model addresses the shortcomings of the past by applying a cross-sectoral approach and multidimensional assessment which allows for strategic and comprehensive planning. The author clarifies that while the MMC is based on communication, it also addresses issues and 
challenges across different sectors and dimensions of development. The first three dimensions (political, organizational, sociocultural) provide the social environmental context assessing gaps and opportunities beyond communication, while the fourth dimension (individual) is where communication methods and techniques are devised to directly promote the intended behavior change. The MMC when driven by C4D is more effective in the development mode, even if it could also be used in the other modes such as emergencies and empowerment. In order to better understand the ways in which the MMC works and how it could help manage the overall planning strategy in C4D projects, the author describes a simulated case study based on a mix of real experiences, which is presented to illustrate how the model could be applied in practice.

In Chapter 13, Guy Bessette explores sustainable development outcomes in agricultural and natural resource management. He posits that many effective agriculture, environment and natural resource management projects tend to be those in which community members, development practitioners or agriculture experts jointly identify research or development parameters and participate in the decision-making process. He reiterates that in these projects, as van de Fliert informed us in Chapter 8, community engagement, consultation and participation occur from the very beginning and continue through the arc of the project. Here, the research and development process itself generates a situation of empowerment in which participants transform their view of reality enabling them to take effective action. This chapter delves deep into participatory development communication (PDC), a branch of the development communication tree that takes a stand on empowerment as the key to sustainable development. The chapter reviews conceptual models associated with PDC, and describes a few cases related to agriculture and natural resource management in Uganda, Burkina Faso and Vietnam where the approach has been used to address development challenges encountered by local communities.

Pradeep Krishnatray, Srinivas Melkote and Leslie Steeves, the authors of Chapter 14, use the COVID-19 pandemic as a case to examine mitigating behaviors and the lack of compliance of many individuals with the recommended safe practices. They examine the reasons for risky behaviors by individuals by reviewing behavior change research, with special reference to psychosocial theory-based behavior change communication (BCC) models. The authors then examine limitations of health communication research in terms of not examining cultural, social and other systemic processes that may impede the adoption of new and safe behaviors by individuals. Many of these structural and cultural factors require a detailed and critical examination as their negative effects are unequally distributed, with the poor and the vulnerable facing the brunt. The authors conclude by offering a normative framework, which posits a role and place for health communication research in inclusive development and progressive social change with social justice as an important anchor.

In Chapter 15, Rafael Obregon and Charlotte Lapsansky examine capacity-building efforts at the organizational level, especially those in C4D and health areas. They echo similar arguments made in Chapters 2 and 3 of the importance of capacity building of individuals. The authors state that strengthening capacities in C4D and health communication is critical to achieving the SDGs and other global health and development goals, particularly during pandemics such as COVID-19. Obregon and Lapsansky present case studies of recent initiatives undertaken by the United Nations Children's Fund (UNICEF) to demonstrate how the organization has evolved a multi-prong and multi-level approach to capacity development, both within its own institution as well as among its partners and governments. The authors review key capacity development concepts and approaches in the literature and analyse UNICEF's 
efforts using a capacity development framework developed for the health sector by Crisp, Swerissen and Duckett (2011) to classify the different approaches of capacity development used by UNICEF and understand the measurement areas to assess their progress. The chapter concludes with a reflection on lessons learned from UNICEF's experience that the authors hope will be of relevance to other institutions and a set of recommendations for policy and practice related to capacity development for C4D and health communication.

\section{Part II: Community and Media Mobilization for Development and Social Change}

The second part of the Handbook covers the role and place of devcom in engaging communities and working to mobilize the community using appropriate media, communication tools and approaches. It includes chapters that describe movements, projects and programs dealing with media and communication in the process of critical social change as well as scholarly critiques of projects and programs in the field of communication and progressive social change.

Emrakeb Woldearegay, Elinam Amevor and Leslie Steeves (Chapter 16) start off this part on gender equity and maternal health promotion in sub-Saharan Africa using participatory and intersectionality approaches. However, the authors caution that this can only succeed when intended recipients have full voice in the definition, design and implementation of programs. This chapter contributes to other critiques of the patriarchal legacy of the modernist approach to healthcare programs in post-colonial Africa, which perpetuates the gender-power relations that marginalize women and girls in the design and implementation of most projects, specifically in sub-Saharan Africa. Within the context of primary healthcare in development, the authors focus on healthcare related to maternal health. The authors discuss maternal healthcare in the contexts of the UN's MDGs and SDGs programs. They focus the discussion on the state of maternal health in sub-Saharan Africa, with Ghana and Ethiopia as case studies. The authors critically examine gender equity in development and maternal health in sub-Saharan Africa and critically assess the African Union's (AU) ambitious campaign to 'end preventable maternal deaths by 2030' and argue why the AU should abide by global equity principles, which require that women and men have equal opportunities for healthcare. The chapter concludes by drawing on participatory and intersectionality approaches to development and social change to argue that the AU should employ these concepts in policy and action decisions to ensure the success of maternal health programs on the African continent.

In Chapter 17, Ami Sengupta and Arvind Singhal illustrate the principles and processes of gender transformative communication by presenting a case study of a long-running social change initiative spearheaded by Minga Perú, a community-based organization in the Peruvian Amazon. The authors apply a gender and power lens and illustrate how media and communication transform existing gender structures. They analyse the monumental shifts that are occurring in roles, norms, hierarchies and relationships between women and men in the Peruvian Amazon emanating from Minga's gender transformative approach. The analysis highlights gendered shifts at the individual, family, community and institutional levels. Through their analysis, Sengupta and Singhal outline a communicative process for gender transformation, highlighting how the Minga approach contributes to multiple gender-related outcomes across health, violence, participation, agency and decision-making. Minga's approach illustrates how the transformation process is inherently communicative. Thus, the authors demonstrate how communication is both a means and an end to achieving gender transformation and broader social change in the community. 
Wendy Papa, Michael Papa and Tisha Dejmanee (Chapter 18) examine the emergence of social media as a central component of social movements to mobilize participants, disseminate messages and generate digitally based activism and protest. This chapter concerns Christine Blasey Ford who came forward with public allegations of sexual assault against US Supreme Court nominee, Judge Brett Kavanaugh in 2018. Dr. Ford was embraced by the bourgeoning online hashtag movement, \#MeToo, and in response to this movement's support for Dr. Ford and opposition to the Kavanaugh nomination, \#HimToo was employed by groups who rejected Dr. Ford's claims. They argued that it was Kavanaugh who was the real victim. The authors conducted a field study by examining publicly posted \#MeToo and \#HimToo tweets in the days leading up to the congressional testimony of Brett Kavanaugh and Christine Ford during Kavanaugh's confirmation hearing. This research describes and analyses theoretical discourses on organizing for social change that were originally formulated through an analysis of field-based movements. The authors contend that as social media become increasingly integrated within social movements to mobilize participants, disseminate messages and generate digitally based activism and protest, it is important to consider the ways that these dialectical discourses might be applicable to contemporary hybrid and digital social movement formations. This study demonstrates a way in which traditional organizational theories may be applied to the study of digital social movements that may be relevant for further inquiries into this growing research area.

In Chapter 19, Uttaran Dutta argues passionately that information poverty and the digital divide, along with structural and communicative absences, including illiteracy, linguistic, cultural, infrastructural, technological, educational and skill barriers, among others, cause discursive disparities as well as erosion of cultural expressions and knowledge in the underserved global South. Dutta posits that top-down interventions to bridge digital divides remain less effective in marginalized communities.

Dutta conducted several CDSC research projects in geographically remote and rural parts of India with the purpose of creating access to information and preserving local cultural resources, and espousing participatory, bottom-up and co-creative visual and sensory approaches, including a visual design (non-digital) intervention in Maharashtra state, and two computer-aided communication design interventions in the state of West Bengal. Eschewing colonial, Western and elite-dominated knowledge production practices, his research as well as other CDSC initiatives have envisioned indigenous participants as active co-researchers and leaders while ensuring dependency reduction, capacity building and sustained transformation. Usage of local languages and visuals, harnessing indigenous wisdom, designing in situ and intercultural competences were some of the key aspects of the communication design initiatives employed in these communities.

In Chapter 20, Shereen Usdin, Nthombahlanga Mqushulu, Lebohang Letsela, Mari Lotvonen and Matokgo Makutoane complete the circle by describing the place and role of communicative actions in the process of critical social change in South Africa. The authors argue forcefully that "exclusion of gender perspectives and obstacles to 'womxn's ${ }^{4}$ participation in public and civic roles impact negatively on democratic principles and good governance. A rights-based model of good governance that is gendered will bring about a more just and equal society." The authors describe a case study on feminist movement building in South Africa by examining the "Raising Young 'womxn's' Voices" project of the Soul City Institute that was conceptualized and implemented to address the marginalization and exclusion of "womxn" and young girls from the nation's political processes. The authors describe how at 
the center of this feminist movement has been the RISE Adolescent Girls and Young "womxn" program and RISE Clubs, an organized movement that builds social cohesion among members, strengthening their capacity to understand and claim their sexual and reproductive health and human rights as well as support their social and economic development. Through mentoring and training, RISE members embarked on civic actions in a coordinated manner, holding accountable municipal and provincial officials. An evaluation of the program showed the potentiality of this feminist movement to overcome the current obstacles to the public participation of "womxn" in provincial and local government processes.

\section{Part III: Conclusion}

We conclude with a chapter in which we draw lessons from the contributions in this book. Key ideas include enhanced role for devcom and participatory communication, active agency of stakeholders of development programs and the operationalization of social justice in development as comprising the twin freedoms of "freedom from inequality" and "freedom for self-actualization" of individuals.

\section{NOTES}

1. Several labels have been used to describe the poorer and industrially underdeveloped countries of the world such as: "Third World," "developing countries" and "less-developed countries."

2. This case draws upon Singhal and Rogers (2003).

3. Descriptions of chapters in this section were provided by the authors through their chapter abstracts.

4. The spelling of "woman" with an " $x$ " is used especially in intersectional feminism, as an alternative spelling to avoid the suggestion of sexism perceived in the sequences m-a-n and m-e-n, and to be inclusive of trans women and nonbinary people (http://www.dictionary.com).

\section{REFERENCES}

Crisp, B., H. Swerissen and S. Duckett (2011), 'Four approaches to capacity building in health: Consequences for measurement and accountability,' Health Promotion International, 15(2), 99-107. Economist (The) (2012), 'For richer, for poorer: Special Report on World Economy,' October 13.

Kemmis, S. (2006), 'Exploring the relevance of critical theory for action research: Emancipatory action research in the footsteps of Jurgen Habermas,' in P. Reason and H. Bradbury (eds.), Handbook of action research, 94-105, London: Sage.

Piketty, T. (2014), Capital in the twenty-first century, Boston, MA: Harvard College.

Sen, A. (2000), Development as freedom, New York: Oxford University Press.

Singhal, A., and E.M. Rogers (2003), Combatting AIDS: Communication strategies in action, Thousand Oaks, CA: Sage.

Stiglitz, J. (2012), The price of inequality, New York: W.W. Norton and Company. 